\title{
ANGULAR ESTIMATES OF CERTAIN INTEGRAL OPERATORS
}

\author{
NAK EUN CHO AND IN HWA KIM
}

Abstract. In the present paper, we investigate certain integral preserving properties in a sector. Our results include several previous results as the special cases.

\section{Introduction}

Let $\mathcal{A}$ denote the class of functions of the form

$$
f(z)=z+\sum_{n=2}^{\infty} a_{n} z^{n}
$$

which are analytic in the open unit disk $U=\{z:|z|<1\}$. A function $f$ of $\mathcal{A}$ is said to be in the class $\mathcal{S}^{*}(\alpha)$, the class of starlike functions of order $\alpha$, if

$$
\operatorname{Re}\left\{\frac{z f^{\prime}(z)}{f(z)}\right\}>\alpha(z \in U, 0<\alpha \leq 1) .
$$

The class $\mathcal{S}^{*}$ of starlike functions is identified by $\mathcal{S}^{*}(0)=\mathcal{S}^{*}$. A function $f \in \mathcal{A}$ is said to be in the class $\mathcal{S}(m, M)$ if

$$
\left|\frac{z f^{\prime}(z)}{f(z)}-m\right|<M(z \in U,|m-1|<M \leq m) .
$$

The class $\mathcal{S}(m, M)$ was introduced by Jakubowski [5]. It is clear that $m>\frac{1}{2}$ and $\mathcal{S}(m, M) \subset \mathcal{S}^{*}(m-M) \subset \mathcal{S}^{*}$.

A function $f \in \mathcal{A}$ is said to be in the class $\mathcal{B}(\mu, \alpha, \beta)$ if it satisfies

$$
\operatorname{Re}\left\{\frac{z f^{\prime}(z) f^{u-1}(z)}{g^{\mu}(z)}\right\}>\beta(z \in U)
$$

for some $\mu(\mu>0), \beta(0 \leq \beta<1)$ and $g \in \mathcal{S}^{*}(\alpha)$. Furthermore, we denote $\mathcal{B}_{1}(\mu, \alpha, \beta)$ by the subclass of $\mathcal{B}(\mu, \alpha, \beta)$ for $g(z) \equiv z \in \mathcal{S}^{*}(\alpha)$. The classes $\mathcal{B}(\mu, \alpha, \beta)$ and $\mathcal{B}_{1}(\mu, \alpha, \beta)$ are the subclasses of Bazilević functions in $U$ [14]. We also note that $\mathcal{B}(1, \alpha, \beta) \equiv C(\alpha, \beta)$ is an important subclass of close-to-convex functions [6].

Received September 18, 1997.

1991 Mathematics Subject Classification. 34C45.

Key words and phrases. Argument, integral operators, starlike functions, Bazilević functions. 
Many authors $[1,2,3,4,8]$ have studied the integral operators of the form

$$
I_{c, \mu}(f)=\left(\frac{c+\mu}{z^{c}} \int_{0}^{z} t^{c-1} f^{\mu}(t) d t\right)^{\frac{1}{\mu}}
$$

where $c$ and $\mu$ are suitably chosen real constants and $f$ belong to some favoured classes of univalent functions. In particular, Kumar and Shukla [8] showed that the integral operator $I_{c, \mu}(f)$ defined by (1.2) maps $\mathcal{S}(m, M)$ into itself for $c \geq-\mu(m-M)(\mu>0)$.

In the present paper, we give some argument properties of the integral operator defined by (1.2). We also generalize the previous results of Libera [9], Owa and Srivastava [12] and Owa and Obradović [13].

\section{Main Results}

In proving our main results, we shall need the following lemmas.

Lemma $1([10])$. Let $M(z)$ and $N(z)$ be regular in $U$ with $M(0)=N(0)=0$, and let $\beta$ be real. If $N(z)$ maps $U$ onto a (possibly many-sheedted) region which is starlike with respect to the orign, then

$$
\operatorname{Re}\left\{\frac{M^{\prime}(z)}{N^{\prime}(z)}\right\}>\beta(z \in U) \Rightarrow \operatorname{Re}\left\{\frac{M(z)}{N(z)}\right\}>\beta(z \in U)
$$

and

$$
\operatorname{Re}\left\{\frac{M^{\prime}(z)}{N^{\prime}(z)}\right\}<\beta(z \in U) \Longrightarrow \operatorname{Re}\left\{\frac{M(z)}{N(z)}\right\}<\beta(z \in U)
$$

Lemma 2([11]). Let $p(z)$ be analytic in $U, p(0)=1, p(z) \neq 0$ in $U$ and suppose that there exists a point $z_{0} \in U$ such that

$$
|\arg p(z)|<\frac{\pi \beta}{2} \text { for }|z|<\left|z_{0}\right|
$$

and

where $0<\beta \leq 1$. Then we have

$$
\left|\arg p\left(z_{0}\right)\right|=\frac{\pi \beta}{2}
$$

$$
\frac{z_{0} p^{\prime}\left(z_{0}\right)}{p\left(z_{0}\right)}=i k \beta
$$

where

$$
k \geq \frac{1}{2}\left(a+\frac{1}{a}\right) \text { when } \arg p\left(z_{0}\right)=\frac{\pi \beta}{2}
$$

and

$$
k \leq-\frac{1}{2}\left(a+\frac{1}{a}\right) \text { when } \arg p\left(z_{0}\right)=-\frac{\pi \beta}{2}
$$


where

$$
p\left(z_{0}\right)^{\frac{1}{\beta}}= \pm i a(a>0) .
$$

Lemma $3([7,8])$. The function $f$ of the form (1.1) belongs to $\mathcal{S}(m, M)$ if and only if there exists a function $w$ regular in $U$ and satisfying $w(0)=0,|w(z)|<1$ for $z \in U$ such that

$$
\frac{z f^{\prime}(z)}{f(z)}=\frac{1+a w(z)}{1-b w(z)} \quad(z \in U)
$$

where $a=\left(M^{2}-m^{2}+m\right) / M$ and $b=(m-1) / M$.

With the help of Lemma 1 and Lemma 2, we now derive

Theorem 1. Let $c$ and $\mu$ be real numbers with $c \geq 0, \mu>0$ and let $f \in \mathcal{A}$. If

$$
\left|\arg \left(\frac{z f^{\prime}(z) f^{\mu-1}(z)}{g^{\mu}(z)}-\beta\right)\right|<\frac{\pi \delta}{2} \quad(0 \leq \beta<1,0<\delta \leq 1)
$$

for some $g \in \mathcal{S}^{*}(m, M)$, then

$$
\left|\arg \left(\frac{z\left(I_{c, \mu}(f)\right)^{\prime} I_{c, \mu}^{\mu-1}(f)}{I_{c, \mu}^{\mu}(g)}-\beta\right)\right|<\frac{\pi \delta}{2}
$$

where $I_{c, \mu}$ is the integral operator defined by (1.2) and $\eta(0<\eta \leq 1)$ is the solution of the equation

$$
\delta=\eta+\frac{2}{\pi} \operatorname{Tan}^{-1}\left(\frac{\eta \sin \frac{\pi}{2}\left(1-\frac{2}{\pi} \sin ^{-1}\left(\frac{M}{c+m}\right)\right)}{c+m+M+\eta \cos \frac{\pi}{2}\left(1-\frac{2}{\pi} \sin ^{-1}\left(\frac{M}{c+m}\right)\right)}\right) .
$$

Proof. Let

$$
p(z)=\frac{M(z)}{N(z)}
$$

where

$$
M(z)=\frac{1}{1-\beta}\left\{z^{c} f^{\mu}(z)-c \int_{0}^{z} t^{c-1} f^{\mu}(t) d t-\beta \mu \int_{0}^{z} t^{c-1} g^{\mu}(t) d t\right\}
$$

and

$$
N(z)=\mu \int_{0}^{z} t^{c-1} g^{\mu}(t) d t
$$

Then $p(z)$ is analytic in $U$ with $p(0)=1$. By a simple calculation, we have

$$
\begin{aligned}
\frac{M^{\prime}(z)}{N^{\prime}(z)} & =p(z)\left(1+\frac{N(z)}{z N^{\prime}(z)} \frac{z^{\prime} p(z)}{p(z)}\right) \\
& =\frac{1}{1-\beta}\left(\frac{z f^{\prime}(z) f^{\mu-1}(z)}{g^{\mu}(z)}-\beta\right) .
\end{aligned}
$$

Since $g \in \mathcal{S}(m, M), I_{c, \mu}(g) \in \mathcal{S}(m, M)$ [8] and hence $N(z)$ is (possibly many sheeted) starlike function with respect to the orign. Therefore, from our assumption and Lemma $1, p(z) \neq 0$ in $U$. 
If there exists a point $z_{0} \in U$ such that

$$
|\arg p(z)|<\frac{\pi \eta}{2} \text { for }|z|<\left|z_{0}\right|
$$

and

then, from Lemma 2, we have

$$
\left|\arg p\left(z_{0}\right)\right|=\frac{\pi \eta}{2}
$$

$$
\frac{z_{0} p^{\prime}\left(z_{0}\right)}{p\left(z_{0}\right)}=i k \eta
$$

where

$$
k \geq \frac{1}{2}\left(a+\frac{1}{a}\right) \text { when } \arg p\left(z_{0}\right)=\frac{\pi \eta}{2}
$$

and

$$
k \leq \frac{1}{2}\left(a+\frac{1}{a}\right) \text { when } \arg p\left(z_{0}\right)=-\frac{\pi \eta}{2}
$$

where

$$
p\left(z_{0}\right)^{\frac{1}{\eta}}= \pm i a(a>0) .
$$

Since $I_{c, \mu}(g) \in \mathcal{S}(m, M)$, we have

$$
\frac{z N^{\prime}(z)}{N(z)}=\frac{z\left(I_{c, \mu}(g)\right)^{\prime}}{I_{c, \mu}(g)}+c=\rho e^{i \frac{\pi \phi}{2}}
$$

where

$$
\left\{\begin{array}{l}
c+m-M<\rho<c+m+M, \\
-\frac{2}{\pi} \sin ^{-1}\left(\frac{M}{c+m}\right)<\phi<\frac{2}{\pi} \sin ^{-1}\left(\frac{M}{c+m}\right) .
\end{array}\right.
$$

At first, suppose that $p\left(z_{0}\right)^{\frac{1}{n}}=i a(a>0)$. We obtain

$$
\begin{aligned}
& \arg \left(\frac{z_{0} f^{\prime}\left(z_{0}\right) f^{\mu-1}\left(z_{0}\right)}{g^{\mu}\left(z_{0}\right)}-\beta\right)=\arg \frac{(1-\beta) M^{\prime}\left(z_{0}\right)}{N^{\prime}\left(z_{0}\right)} \\
& =\arg p\left(z_{0}\right)+\arg \left(1+\frac{1}{\frac{z\left(I_{c, \mu}(g)\right)^{\prime}}{I_{c, \mu}(g)}+c} \frac{z_{0} p^{\prime}\left(z_{0}\right)}{p\left(z_{0}\right)}\right) \\
& =\frac{\pi \eta}{2}+\arg \left(1+\left(\rho e^{i \frac{\pi \phi}{2}}\right)^{-1} i \eta k\right) \\
& =\frac{\pi \eta}{2}+\operatorname{Tan}^{-1}\left(\frac{\eta k \sin \frac{\pi}{2}(1-\phi)}{\rho+\eta k \cos \frac{\pi}{2}(1-\phi)}\right) \\
& \geq \frac{\pi \eta}{2}+\operatorname{Tan}^{-1}\left(\frac{\eta \sin \frac{\pi}{2}\left(1-\frac{2}{\pi} \sin ^{-1}\left(\frac{M}{c+m}\right)\right)}{\left.c+m+M+\eta \cos \frac{\pi}{2}-11-\frac{2}{\pi} \sin ^{-1}\left(\frac{M}{c+m}\right)\right)}\right) \\
& =\frac{\pi}{2} \delta,
\end{aligned}
$$

where $\delta$ are given by (2.2). This is a contradiction to the assumption of our theorem. 
Next, suppose that $p\left(z_{0}\right)^{\frac{1}{n}}=-i a(a>0)$. Applying the same method as the above, we have

$$
\begin{aligned}
\arg \left(\frac{z_{0} f^{\prime}\left(z_{0}\right) f^{\mu-1}\left(z_{0}\right)}{g^{\mu}\left(z_{0}\right)}-\beta\right) & \leq-\frac{\pi \eta}{2}-\operatorname{Tan}^{-1}\left(\frac{\eta \sin \frac{\pi}{2}\left(1-\frac{2}{\pi} \sin ^{-1}\left(\frac{M}{c+m}\right)\right)}{c+m+M+\eta \cos \frac{\pi}{2}\left(1-\frac{2}{\pi} \sin ^{-1}\left(\frac{M}{c+m}\right)\right)}\right) \\
& =-\frac{\pi}{2} \delta
\end{aligned}
$$

where $\delta$ are given by (2.2), which contracts the assumption. Therefore we complete the proof of our theorem.

Let us choose $m=N-\alpha(N-1)$ and $M=N(1-\alpha)$, where $N \geq 1$ and $0 \leq \alpha<1$. Then $|m-1|<M \leq m, a=\alpha / N+(1-2 \alpha)$ and $b=1-1 / N$ in Lemma 3. Now as $N \rightarrow \infty, a \rightarrow 1-2 \alpha$ and $b \rightarrow 1$. In this case, the relation (2.1) reduces to

$$
\frac{z f^{\prime}(z)}{f(z)}=\frac{1+(1-2 \alpha) w(z)}{1-w(z)}(z \in U)
$$

which is a necessary and sufficient condition for $f$ to be in $\mathcal{S}^{*}(\alpha)$. Hence we have the following

Corollary 1. Let $c \geq 0, \mu>0$ and $f \in \mathcal{A}$. If

$$
\left|\arg \left(\frac{z f^{\prime}(z) f^{\mu-1}(z)}{g^{\mu}(z)}-\beta\right)\right|<\frac{\pi \delta}{2} \quad(0 \leq \beta<1,0<\delta \leq 1)
$$

for some $g \in \mathcal{S}^{*}(\alpha)$, then

$$
\left|\arg \left(\frac{z\left(I_{c, \mu}(f)\right)^{\prime} I_{c, \mu}^{\mu-1}(f)}{I_{c, \mu}^{\mu-1}(g)}-\beta\right)\right|<\frac{\pi \delta}{2}
$$

where $I_{c, \mu}$ is the integral operator defined by (1.2).

Remark $\mathbb{1}$. For $\delta=1$, Corollary 1 is the result obtained by Owa and Obradović [13].

Letting $\mu=1$ in Theorem 1 , we have

Corollary 2. Let $c \geq 0$ and let $f \in \mathcal{A}$. If

$$
\left|\arg \left(\frac{z f^{\prime}(z)}{g(z)}-\beta\right)\right|<\frac{\pi \delta}{2} \quad(0 \leq \beta<1,0<\delta \leq 1)
$$

for some $g \in \mathcal{S}(m, M)$, then

$$
\left|\arg \left(\frac{z\left(I_{c, 1}(f)\right)^{\prime}}{I_{c, 1}(g)}-\beta\right)\right|<\frac{\pi \eta}{2}
$$

where $I_{c, 1}$ is the integral operator defined by (1.2) and $\eta(0<\eta \leq 1)$ is the solution of the equation (2.2). 
Taking $m=N-\alpha(N-1), M=N(1-\alpha)(0 \leq \alpha<1), N \rightarrow \infty$ and $\delta=1$ in Corollary 2 , we have the result of Owa and Srivastave [12].

Corollary 3. If the function $f$ defined by (1.1) is in the class $\mathcal{C}(\alpha, \beta)$, then the integral operator $I_{c, 1}(f)(c \geq 0)$ defined by $(1.2)$ is also in the class $\mathcal{C}(\alpha, \beta)$.

Remark 2. Putting $\alpha=\beta=0$ and $c=1$ in Corollary 3, we obtain the result given by Libera [9].

By using the same technique as in proving Theorem 1, we have

Theorem 2. Let $c$ and $\mu$ be real numbers with $c \geq 0, \mu>0$ and let $f \in \mathcal{A}$. If

$$
\left|\arg \left(\beta-\frac{z f^{\prime}(z) f^{\mu-1}(z)}{g^{\mu}(z)}\right)\right|<\frac{\pi \delta}{2} \quad(\beta>1,0<\delta \leq 1)
$$

for some $g \in \mathcal{S}(m, M)$, then

$$
\left|\arg \left(\beta-\frac{z\left(I_{c, \mu}(f)\right)^{\prime} I_{c, \mu}^{\mu-1}(f)}{I_{c, \mu}^{\mu}(g)}\right)\right|<\frac{\pi \eta}{2},
$$

where $I_{c, \mu}$ is the integral operator defined by (1.2) and $\eta(0<\eta \leq 1)$ is the solution of the equation (2.2).

Letting $m=N-\alpha(N-1), M=N(1-\alpha)(0 \leq \alpha<1), N \rightarrow \infty, \mu=1$ and $\delta=1$ in Theorem 2, we have the following result by Owa and Srivastava [12].

Corollary 4. Let $c \geq 0$ and $f \in \mathcal{A}$. If

$$
\operatorname{Re}\left\{\frac{z f^{\prime}(z)}{g(z)}\right\}<\beta \quad(\beta>1)
$$

for some $g \in \mathcal{S}^{*}(\alpha)$, then

$$
\operatorname{Re}\left\{\frac{z\left(I_{c, 1}(f)\right)^{\prime}}{I_{c, 1}(g)}\right\}<\beta
$$

where $I_{c, 1}$ is the integral operator defined by (1.2).

\section{Acknowledgement}

This work was partially supported by Non Directed Research Fund, Korea Research Foundation, 1996 and the Basic Science Research Program, Ministry of Education, Project No. BSRI-97-1440. 


\section{References}

[1] G. P. Bhargave and S. L. Shukla, "On the classes of regular univalent functions," Jnanabha, 12(1982), 33-39.

[2] W. M. Cauchy and W. L. White, "Starlikeness of certain functions with integral representations," J. Math. Anal. Appl., 64(1978), 458-466.

[3] R. M. Goel and B. S. Mehrok, "Some invarance properties of a subclass of close-to-convex functions," Indian J. Pure. Appl. Math., 12(1981), 1240-1249.

[4] V. P. Gupta and P. K. Jain, "On starlike functions," Rend. Mat., 9(6)(1976), 433-437.

[5] Z. J. Jakubowski, "On the coefficients of starlike functions of some classes," Ann. Polon. Math., 26(1972), 305-313.

[6] W. Kaplan, "Close-to-convex schlicht functions," Michigan Math. J., 1(1952), 169-185.

[7] V. Kumar and S. L. Shukla, "Certain integrals for classes of $p$-valent meromorphic functions," Bull. Austral. Math. Soc., 25(1982), 85-97.

[8] V. Kumar and S. L. Shukla, "Jakubowski integral operators," J. Austral. Math. Soc. (series A), 37(1984), 117-127.

[9] R. J. Libera, "Some classes of regular univalent functions," Proc. Amer. Math. Soc., 16(1965), $755-758$.

[10] S. S. Miller and P. T. Mocanu, "Second order differential inequalities in the complex plane," J. Math. Anal. Appl., 65(1978), 289-305.

[11] M. Nunokawa, "On the order of strongly starlikeness of strongly convex functions," Proc. Japan Acad., 69, Ser. A(1993), 234-237.

[12] S. Owa and H. M. Srivastava, "Some applications of the generalized Libera integral operator," Proc. Japan Acad., 62, Ser. A(1986), 125-128.

[13] S. Owa and M. Obradović, "Certain subclasses of Bazilević functions of type $\alpha$," Internat. J. Math. \& Math. Sci., 9(1986), 347-359.

[14] R. Singh, "On Bazilević functions," Proc. Amer. Math. Soc., 1(1952), 169-185.

Department of Applied mathematics, College of Natural Sciences, Pukyong National University, Pusan 608-737, Korea. 